\title{
Effects of indigo naturalis on colonic mucosal injuries and inflammation in rats with dextran sodium sulphate-induced ulcerative colitis
}

\author{
YUNLIANG WANG $^{1 *}$, LIJUAN LIU ${ }^{2 *}$, YI GUO $^{1,3}$, TANGYOU MAO $^{1,3}$, RUI SHI $^{1}$ and JUNXIANG LI ${ }^{1}$ \\ ${ }^{1}$ Department of Gastroenterology, Dongfang Hospital, Beijing University of Chinese Medicine, Beijing 100078; \\ ${ }^{2}$ Gastroenterology Department of Traditional Chinese Medicine, China-Japan Friendship Hospital; \\ ${ }^{3}$ Graduate School, Beijing University of Chinese Medicine, Beijing 100029, P.R. China
}

Received February 21, 2016; Accepted March 17, 2017

DOI: 10.3892/etm.2017.4701

\begin{abstract}
The effects of indigo naturalis (IN), which is a traditional Chinese herbal formulation, have been clinically demonstrated in treating refractory ulcerative colitis (UC). The present study aimed to verify the effects and mechanisms of IN in experimental UC rats. A total of 48 male Sprague-Dawley rats were randomly divided into six groups: Chow, model, high-dose IN, medium-dose IN, low-dose IN and mesalazine (a bowel-specific aminosalicylate drug) groups. The models were administered $3.5 \%$ dextran sodium sulphate solution for 7 days. The treatment groups were administered IN or mesalazine and then sacrificed and sampled on day 8. Disease activity index (DAI), histological damage score (HDS) and myeloperoxidase (MPO) activity were used to evaluate the severity of UC. Colon and serum cytokines were detected using liquid-phase chip technology and the expression of occludin protein in colonic mucosa was assessed by immunohistochemistry and western blot analysis. The results indicated that the oral administration of IN may reduce DAI, HDS and MPO activity. IN also reduced the expression of inflammatory cytokines and increased the expression of colonic mucosal repair-related cytokines and occludin protein. These results highlight the potential of IN as a therapeutic agent for treating UC through its action of inflammation control and colonic mucosal damage repair.
\end{abstract}

Correspondence to: Dr Junxiang Li, Department of Gastroenterology, Dongfang Hospital, Beijing University of Chinese Medicine, 6 1st section, Fangxingyuan, Fangzhuang, Beijing 100078, P.R. China

E-mail: lijunxiang1226@163.com

${ }^{*}$ Contributed equally

Key words: indigo naturalis, Chinese herbal medicine, ulcerative colitis, inflammation, colonic mucosal changes

\section{Introduction}

Ulcerative colitis (UC), one of the primary forms of inflammatory bowel disease (IBD), is a non-specific, chronic inflammatory disorder of the colonic mucosa. Its primary features are abdominal pain, diarrhoea, bloody mucopurulent stool and alternating periods of exacerbation and remission of clinical symptoms (1). The incidence and prevalence of UC is increasing globally: The highest annual incidence of UC was 24.3 in 100,000 individuals/year in Europe, 6.3 in 100,000 individuals/year in Asia and the Middle East and 19.2 in 100,000 individuals/year in North America (2). UC tends to persist for long durations, and patients are prone to relapse and exhibit a higher risk of developing colorectal cancer (CRC) than the general population (3). In addition, the detection rate of UC-associated CRC (UC-CRC), one of the most serious complications, has also increased. The probability of CRC in patients with UC increases with the duration of disease; $1.6 \%$ at 10 years after onset, $8.3 \%$ at 20 years and $18.4 \%$ at 30 years after onset (4). Owing to the delayed healing and lifetime debilitating physical symptoms (urgent diarrhoea, rectal bleeding, vomiting, anorexia and lethargy), patients typically suffer poor psychosocial well-being (5).

At present, the precise cause of UC remains poorly understood; however, its pathophysiology has been demonstrated to be a complex result of epithelial barrier defects, commensal microflora, antigen recognition, dysregulation of immunological responses, leukocyte recruitment and genetic factors (1), of which the balance between anti- and pro-inflammatory signals serves a vital role. Furthermore, inflammation resulting from a failure to maintain this balance is evident in patients with immune dysregulation (6).

The CRC involves continuous intestinal inflammation, non-dysplastic mucosa, indefinite dysplasia, low-grade dysplasia, high-grade dysplasia and finally invasive adenocarcinoma (7). Furthermore, the progression from inflammation to atypical hyperplasia to cancer in patients with UC is more rapid than the progression of adenoma to adenocarcinoma in the general population $(8,9)$. Thus, as an independent risk factor, intestinal inflammation is the initial step in UC-CRC development, and the risk of CRC increases with the severity 
of inflammation in patients with atypical hyperplasia or chronic UC. Furthermore, the length of the disease course is a key factor for cancer in patients with UC. The average incidence rate of cancer increases exponentially with the disease lasting $>10$ years (10). Therefore, as the control of inflammation ameliorates UC, to enhance the quality of life and work efficiency, it also prevents the development of CRC and reduces its incidence.

Although research on UC has progressed a great deal, effective treatment approaches remain to be elucidated. The treatment of UC aims to relieve the symptoms and complications, in addition to preventing recurrence and improving the patients' quality of life (11). UC treatment includes glucocorticosteroids, aminosalicylates, immunomodulators, biopharmaceuticals, surgery and diet therapy. Among these, aminosalicylates and glucocorticosteroids are currently the first-line agents for mild-moderate and moderate-severe UC (12), respectively. Use of these agents, however, has a number of problems, including lack of tolerance to the drug, side effects, prolonged treatment duration and high recurrence rates. In addition, the efficacy and safety of immunomodulators and antibiotics, which are currently in the clinical trial stages, require further evaluation (13). Therefore, there is an increasing requirement for the development of more effective and less toxic agents for UC treatment.

Indigo naturalis (IN), which is a traditional Chinese medicinal composition comprising the dried powder of processed leaves or stems of Baphicacanthus cusia (Nees) Bremek, Polygonum tinctorium or Isatis indigotica Fort, has been demonstrated to be effective in treating UC (14). The primary active ingredients are indigo, indirubin, tryptanthrin, and qingdainone. The clinical disease activity indexes (DAIs) and endoscopic Matts grades have been demonstrated to significantly decrease following oral administration of IN powder in patients with UC who are unresponsive to 5-aminosalicylic acid (5-ASA), prednisolone and infliximab treatment (15). The majority of patients were relieved of hormone dependence. Furthermore, IN has a powerful scavenging effect on hydroxyl radicals in patients with UC. IN extract has been successfully used to treat clinical psoriasis (16) and induce the apoptosis and autophagy of acute lymphoblastic leukaemia cell lines (17).

The present study aimed to provide an experimental basis for the use of IN as a potential therapeutic agent for UC by investigating the possible protective mechanism of IN in dextran sulphate sodium (DSS)-induced UC rats on the basis of colonic mucosal injuries and inflammation. The effects of IN were investigated in comparison with the bowel-specific aminosalicylate drug mesalazine.

\section{Materials and methods}

Preparation of IN and mesalazine slow release (SR) granules. IN granules comprising crude IN were provided by the Pharmacy Department of Dongfang Hospital, Beijing University of Chinese Medicine (Beijing, China). IN was administered orally at high (INH), medium (INM) and low (INL) doses (16.8, 8.4 and $4.2 \mathrm{~g} / \mathrm{kg}$, respectively) of the crude drug (in terms of medicinal material quality in rats). Mesalazine SR granules were provided by Shanghai Ethypharm Pharmaceutical Co., Ltd., (Shanghai, China).
Animals and treatment. A total of 48 healthy male Sprague-Dawley rats (age, 7 weeks; weight, 180-220 g) were supplied by Vital River Laboratories Co., Ltd. [Beijing, China; license no. SCXK (Jing) 2012-0001]. The rats were maintained under a 12-h light/dark cycle under constant temperature of $22 \pm 2^{\circ} \mathrm{C}$ and $50-60 \%$ humidity, with ad libitum access to water. All experimental procedures were approved by the Animal Ethics Committee of Beijing University of Chinese Medicine under the guidelines issued by the Regulations of Beijing Laboratory Animal Management. IN or mesalazine granules were dissolved in $100 \mathrm{ml}$ normal saline and stored at $2-8^{\circ} \mathrm{C}$ until use. The rats were provided ad libitum access to $3.5 \%$ DSS solution (36-50 kDa; MP Biomedical, LLC, Santa Ana, CA, USA) for seven days to prepare the acute experimental UC rat model, as described previously (18). The animals in the INL (4.2 g/kg/day), INM (8.4 g/kg/day), INH (16.8 g/kg/day), and mesalazine (Mes; $400 \mathrm{mg} / \mathrm{kg} /$ day) groups ( $\mathrm{n}=8$ in all groups) were administered the respective doses at the beginning of $3.5 \%$ DSS feeding. Normal saline $(10 \mathrm{ml} / \mathrm{kg} /$ day $)$ was administered orally to chow-fed rats (chow, $n=8$ ) and the model control (model, $\mathrm{n}=8$ ). Chow-fed rats were provided to access to water ad libitum, whereas rats in the model group were provided with ad libitum access to DSS solution. All groups were administered their respective treatment or saline orally for seven days.

Detection of DAI. Rat stool samples were harvested and evaluated every day, and fecal occult blood test papers (BaSO Biotechnology, New Taipei, Taiwan) were used to test for fecal occult blood following the manufacturer's protocol. The scoring method of Hamamoto (19) was used to calculate the rat DAI score and evaluate disease activity. The DAI score includes three aspects: Stool, faecal occult blood, and body weight loss, scored on a 0-4-point system. For body mass, points were distributed as follows; 0 , no drop in body mass; $1,1-5 \%$ drop in body mass; $2,5-10 \%$ drop in body mass; 3 , $10-15 \%$ drop in body mass; and $4,>15 \%$ drop in body mass. For stool, points were distributed as follows; 0, normal stool; 2, loose stool; and 4, diarrhoea. For fecal occult blood, points were distributed as follows; 0, no blood; 2, positive; and 4, naked bloody stool. Normal stool was well formed or shaped; loose stool, not adhered to the anus, pasty, or semi-formed; and diarrhoea, shapeless and adhering to the anus. DAI was evaluated daily in a blinded manner by non-project team members as follows: DAI=(weight loss score+stool consistency score+fecal occult blood score)/3 (20).

Colon pathology analysis in rats. After blood $(10 \mathrm{ml})$ was sampled from the abdominal aorta, all rats were anesthetized with $10 \%$ chloral hydrate $(300 \mathrm{mg} / \mathrm{kg}$, Sinopharm Chemical Reagent Co., Ltd., Shanghai, China) and sacrificed by decapitation. Colon tissues were harvested, fixed in $10 \%$ formalin for $24 \mathrm{~h}$ at $22^{\circ} \mathrm{C}$, embedded in paraffin and cut into $4 \mu \mathrm{m}$ sections. Haematoxylin and eosin staining was performed using paraffin sections. The histological score (HS) was determined by microscopically under a light microscope (Olympus Corp., Tokyo, Japan) examining stained paraffin sections using the scoring criteria of colonic histological activity index (21-23). Each sample was randomly selected from five perspectives to calculate the total score (0-10 points), including the infiltration 
Table I. Histological activity index scoring.

\begin{tabular}{llll}
\hline Score & \multicolumn{1}{c}{ Inflammation } & Ulcer & Layer \\
\hline 0 & A small amount of inflammatory cells in the lamina propria & None & None \\
1 & More neutrophil granulocytes in the lamina propria & $1-2$ ulcers & Mucosa \\
2 & Infiltration of inflammatory cells to the submucosa & $3-4$ ulcers & Submucosa \\
3 & Permeability of inflammatory cells infiltration to the & Wide range or & Muscle \\
4 & whole layers & continuous ulcer & -
\end{tabular}

degree of inflammatory cells, ulcers and lesion level (Table I). The HS assessment was completed by non-project team members under the guidance of a pathologist and blindly reviewed by specific members.

Detection of colon myeloperoxidase activity. Colonic myeloperoxidase (MPO) activity was detected according to a previously described method (24). Colonic tissue was dissected $1 \mathrm{~cm}$ above the anus, washed with ice-cold saline and weighed. Subsequently, $1 \mathrm{ml}$ hexadecyl trimethyl ammonium bromide (HTAB) buffer (0.5\% HTAB 50 mmol; 1 PBS, pH 6.0; Sigma-Aldrich; Merck KGaA, Darmstadt, Germany) was used for tissue homogenisation. The tissue homogenate was centrifuged at $60,000 \mathrm{x}$ g for $15 \mathrm{~min}$ at $4^{\circ} \mathrm{C}$. The absorbance optical density (OD) 1 of $0.1 \mathrm{ml}$ supernatant added with $2.9 \mathrm{ml} 50 \mathrm{mmol} / \mathrm{l}$ phosphate buffered saline (PBS; containing $0.167 \mathrm{mg} / \mathrm{ml} o$-dianisidine and $0.0005 \% \mathrm{H}_{2} \mathrm{O}_{2}$ ) was immediately measured at $460 \mathrm{~nm}$ using a spectrometer (Thermo Fisher Scientific, Inc., Waltham, MA, USA), and the absorbance OD2 was measured 1 min later. MPO activity was calculated as (OD2-OD1)/total protein (OD/mg protein).

Detection of cytokines. The supernatants of fresh colon tissue and serum were carefully prepared and analysed following the protocol of a commercial liquid-phase chip kit (RECYMAG65K04; Merck KGaA). Serum cytokines [interleukin (IL)-1 $\alpha$, IL-1 $\beta$, IL1-18, epidermal growth factor (EGF) and vascular endothelial growth factor] were expressed as $\mathrm{pg} / \mathrm{ml}$, and those in the tissues were expressed as $\mathrm{pg} / \mathrm{mg}$ protein.

Western blot analysis to detect occludin. Proteins in $100 \mathrm{mg}$ colonic tissue homogenate were extracted using ice-cold radioimmunoprecipitation assaay lysis buffer with a final concentration of $1 \mathrm{mM}$ PMSF at $4^{\circ} \mathrm{C}$ in an electric tissue homogenizer (ULTRA-TURRAX; IKA Industrie- und Kraftfahrzeugausrüstung $\mathrm{GmbH}$, Konigswinter, Germany) at $250 \mathrm{x}$ g for $5 \mathrm{sec}, 5$ times at $5 \mathrm{sec}$ intervals, and incubated on ice for $20 \mathrm{~min}$ following homogenization. The supernatant was extracted by centrifugation at $12,000 \mathrm{x} \mathrm{g}$ for $20 \mathrm{~min}$ at $4^{\circ} \mathrm{C}$. Protein concentrations were determined by using a bicinchoninic acid protein assay kit (Promega, Madison, WI, USA). Samples $(30 \mu \mathrm{g})$ were separated by $10 \%$ SDS-PAGE and transferred onto polyvinylidene difluoride membranes. The membranes were then incubated in 5\% skimmed milk powder for $2 \mathrm{~h}$ prior to the addition of primary rabbit anti-occludin polyclonal antibody (ab31721; 1:2,000; Abcam, Cambridge, MA, USA) and GAPDH rat antibody (TDY042, 1:20,000;
Beijing TDY Biotech Co., Ltd., Beijing, China) and incubation at $4^{\circ} \mathrm{C}$ overnight. Membranes were subsequently incubated with peroxidase-conjugated goat anti-rabbit secondary antibody (1:20,000; cat. no. S001; Beijing TDY Biotech, Co., Ltd.) at room temperature for $2 \mathrm{~h}$, and an ECL detection system (horseradish peroxidase substrate; WBKLS0500; EMD Millipore, Billerica, MA, USA) was used according to routine methods (25). The intensities of the protein bands were analysed using Gel-Pro 3.2 software (Media Cybernetics, Inc., Rockville, MD, USA). GAPDH protein was used as the internal control to normalize the protein loading. The experiment was performed in triplicate.

Immunohistochemistry of occludin. Following conventional dehydration, embedding (26) and tissue slicing (thickness, $4 \mu \mathrm{m}$ ) of colon tissues, the paraffin sections were dewaxed and hydrated (26). The diluent primary rabbit anti-human occludin antibody (ab31721; 1:100; Abcam) was used after the tissue antigens were repaired in a high pressure cooker, $>120^{\circ} \mathrm{C}$. Secondary antibody incubation was performed according to the instructions of the PV9000 secary antibody kit (Beijing Zhongshan Golden Bridge Biotechnology Co., Ltd., Beijing, China), followed by 3,3'-diaminobenzidine staining. Five images (mucosal layer) were randomly collected in each tissue slice at a magnification of $\mathrm{x} 400$ under a light microscope for analysis using Image Plus 6.0 software (Media Cybernetics, Inc.). The OD was used to represent protein expression, and the total stained area was used to express the distribution and total expression area of the target protein.

Determination of liver enzymes in rats. An automatic biochemical analyser (CX4 Pro; Beckman Coulter, Inc., Brea, CA, USA) (27) was used to detect serum alanine transaminase (ALT) and aspartate transaminase (AST) to assess the safety of IN.

Statistical analysis. All data are expressed as the mean \pm standard deviation, unless otherwise indicated, and were analysed with SPSS 17.0 statistical software (SPSS, Inc., Chicago, IL, USA). The data that met the normal distribution and homogeneity of variance criteria were subjected to one-way analysis of variance and pairwise comparison was performed with the least significant difference test. The data that fulfilled normal distribution but did not meet the heterogeneity of variance criteria were subjected to Tamhane's T2 test for pairwise comparison. $\mathrm{P}<0.05$ was considered to indicate a statistically 


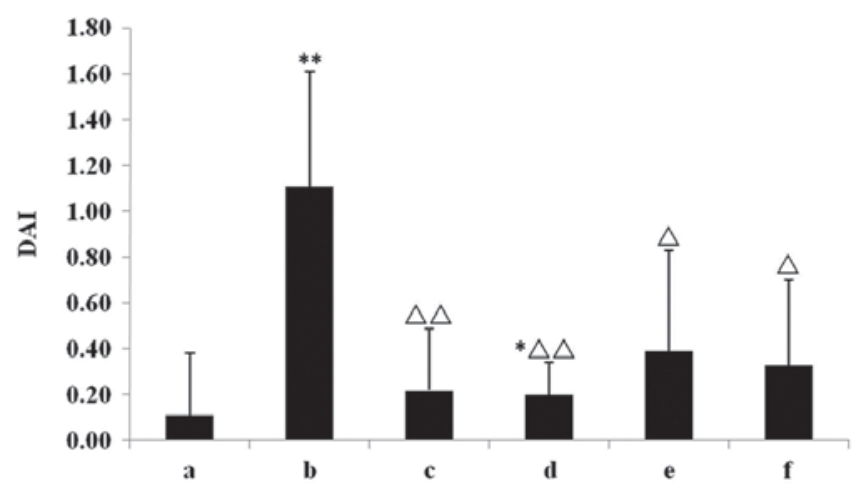

Figure 1. Effect of IN on the detection of DAI. The Mes treatment and all the IN treatments significantly attenuated the elevated DAI. ${ }^{*} \mathrm{P}<0.05,{ }^{* *} \mathrm{P}<0.01$ vs. chow group; ${ }^{\Delta} \mathrm{P}<0.05,{ }^{\Delta \Delta} \mathrm{P}<0.01$ vs. model group. Data are presented as mean \pm standard deviation. IN, indigo naturalis; DAI, disease activity index; Mes, mesalazine; a, chow group; b, model group; c, Mes group; d, IN high-dose group; e, IN medium-dose group; f, IN low-dose group.
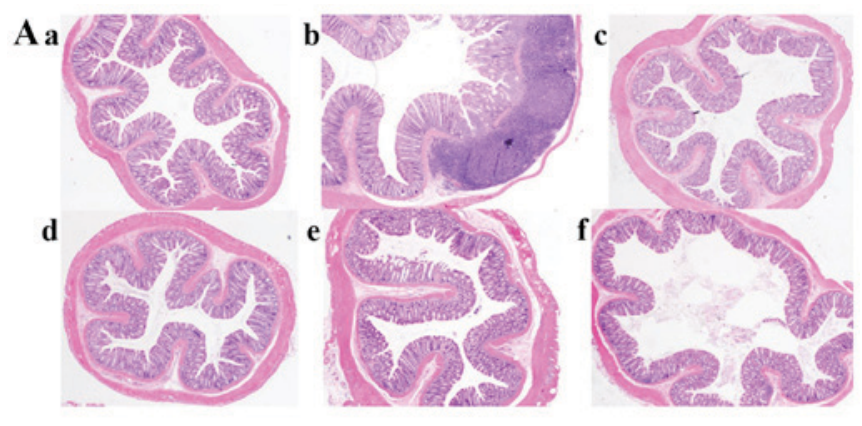

B

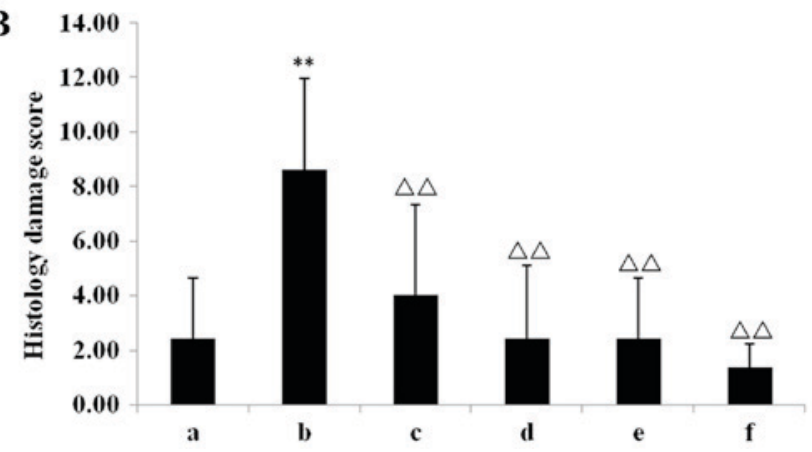

Figure 2. Mucosal histopathology changes in rat. (A) Haematoxylin and eosin stained paraffin sections of the (Aa) chow, (Ab) model, (Ac) Mes, (Ad) IN high-dose, (Ae) IN medium-dose, and (Af) IN low-dose groups. In the dextran sulphate sodium model group, the colonic mucosa indicates congestion, oedema and excessive infiltration of inflammatory cells. Magnification, x40. (B) All treatment groups demonstrate reduced congestion, oedema and infiltration of inflammatory cells. Compared with the chow group, the colonic histopathological score of the model group was significantly higher. The Mes group and all IN groups had lower colonic histopathologic scores compared with the model group. ${ }^{* *} \mathrm{P}<0.01$ vs. chow group; ${ }^{\Delta \Delta} \mathrm{P}<0.01$ vs. model group. Data are presented as mean \pm standard deviation. Mes, mesalazine; IN, indigo naturalis; INH, IN high-dose; INM, IN medium-dose; INL, IN low-dose; a, chow group; b, model group; c, Mes group; d, IN high-dose group; e, IN medium-dose group; f, IN low-dose group.

significant difference. The data that did not meet the normal distribution or homogeneity of variance criteria were subjected to non-parametric testing, with $\mathrm{P}<0.008$ following correction considered to indicate a statistically significant difference.

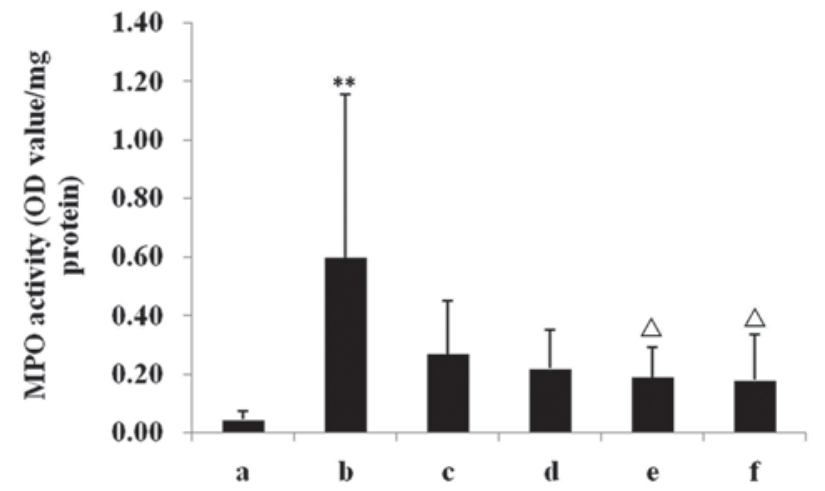

Figure 3. Detection of colon MPO activity. Compared with the chow group, the MPO activity in the colon was significantly higher in the dextran sulphate sodium model group. Both IN medium-dose and IN low-dose groups had low MPO activities. ${ }^{* *} \mathrm{P}<0.01$ vs. chow group; ${ }^{\Delta} \mathrm{P}<0.05$ vs. model group. Data are presented as mean \pm standard deviation. MPO, myeloperoxidase; IN, indigo naturalis; a, chow group; b, model group; c, mesalazine group; d, IN high-dose group; e, IN medium-dose group; f, IN low-dose group.

\section{Results}

DAI. All rats tolerated the experimental procedures well, and none succumbed to mortality during the study. Following ad libitum access to $3.5 \%$ DSS for two days, positive fecal occult blood began to appear in the DSS group; however, no marked change was observed in hair colour or weight (which are indicative of the health of rats). With an increase in DSS consumption, haematochezia was exacerbated and gross blood began to appear in the stool, together with the lack of hair colour lustre, slowing of activity and reaction to stimulus. The maximum DAI score was observed at 7 days. Compared with the chow group, DAI scores in the model group were significantly increased $(\mathrm{P}<0.01$; Fig. 1). Furthermore, Mes treatment and all the IN treatments significantly attenuated the elevated DAI compared with the model group $(\mathrm{P}<0.05$; Fig. 1).

Mucosal histopathology changes in rats. Histological analysis demonstrated few inflammatory cells in the mucosa, and normal crypts and epithelial integrity in the chow group (Fig. 2Aa). In the DSS model group, light microscopy of the colonic mucosa indicated congestion, edema and excessive infiltration of inflammatory cells, which were primarily neutrophils. There were very few goblet cells and the mucosa was continuously broken. Ulceration was observed deep into the submucosa, and the lesions were primarily concentrated from the mucosal layer to the submucosal layer (Fig. 2Ab). Conversely, all treatment groups exhibited reduced congestion, oedema and infiltration of inflammatory cells (Fig. 2Ac-f). Compared with the chow group, the colonic histopathological score of the model group was significantly higher $(\mathrm{P}<0.01$; Fig. 2B). The mesalazine group and all IN groups exhibited significantly reduced colonic histopathologic scores compared with the model group $(\mathrm{P}<0.01$; Fig. $2 \mathrm{~B})$.

Activities of colon MPO. Compared with the chow group, the MPO activity in the colon was significantly higher in the DSS model group $(\mathrm{P}<0.01$; Fig. 3). INM and INL treatments significantly decreased the MPO activities compared with the model 

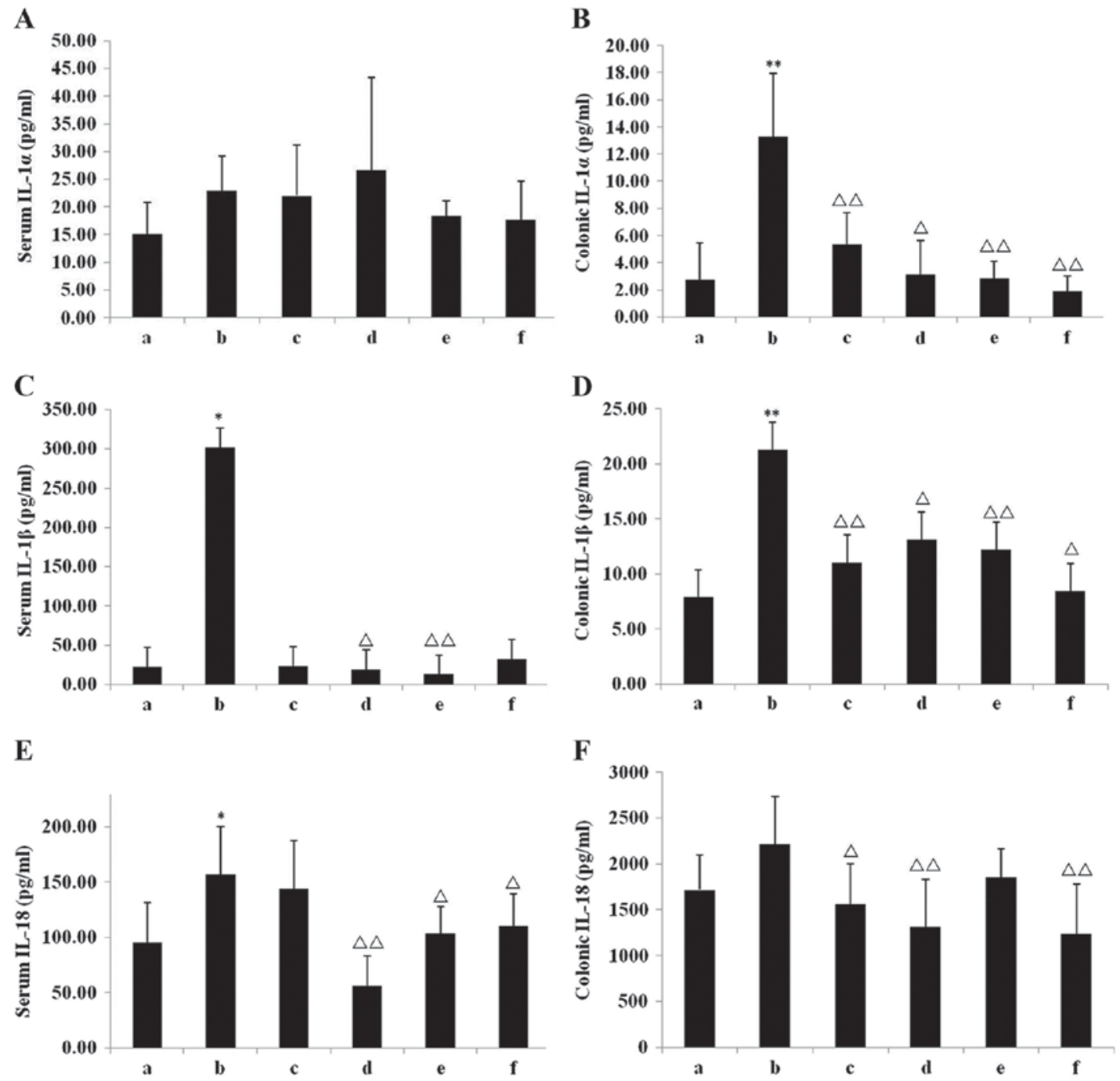

Figure 4. Inflammatory cytokine levels in serum and colonic tissue. (A) Serum IL-1 $\alpha$, (B) colonic IL-1 $\alpha$, (C) serum IL-1 $\beta$, (D) colonic IL-1 $\beta$, (E) serum IL-18, and (F) colonic IL-18 in the 6 groups. ${ }^{*} \mathrm{P}<0.05,{ }^{* *} \mathrm{P}<0.01$ vs. chow group; ${ }^{\wedge} \mathrm{P}<0.05,{ }^{\Delta} \mathrm{P}<0.01$ vs. model group. Data are presented as mean \pm standard deviation. IL, interleukin; a, chow group; b, model group; c, mesalazine group; d, IN high-dose group; e, IN medium-dose group; f, IN low-dose group; IN, indigo naturalis.

group ( $\mathrm{P}<0.05$; Fig. 3). As presented in Fig. 3, although the MPO in INH and Mes groups decreased, this was not statistically significant.

Level of inflammatory cytokines. Compared with the chow group, the level of serum IL-1 $\alpha$ in the model group increased, although this was not statistically significant (Fig. 4A). The level of IL- $1 \alpha$ and IL- $1 \beta$ in the colonic tissue of the model group, however, was increased significantly compared with the chow group $(\mathrm{P}<0.01$; Fig. $4 \mathrm{~B})$, whereas levels of IL-1 $\beta$ were significantly increased in the model group compared with the chow group in both the serum $(\mathrm{P}<0.05$; Fig. $4 \mathrm{C})$ and colonic tissue $(\mathrm{P}<0.01$; Fig. 4D). IL-18 levels were significantly increased in the model group compared with the chow group in the serum $(\mathrm{P}<0.05$; Fig. $4 \mathrm{E})$ and markedly in colonic tissue (Fig. 4F). All IN treatments reduced the level of IL- $1 \alpha$ IL-1 $\beta$ and IL-18 in the colonic tissue. In INH, a significant reduction was observed in the serum IL-1 $\beta(\mathrm{P}<0.05$; Fig. 4C) and IL-18 $(\mathrm{P}<0.01$; Fig. 4E) levels, in addition to colonic IL-1 $\alpha(\mathrm{P}<0.05$; Fig. 4B), IL-1 $\beta$ ( $\mathrm{P}<0.05$; Fig. 4D) and IL-18 (P<0.01; Fig. 4F) levels, in comparison with the model group. In INM, the serum levels of IL-1 $\beta(\mathrm{P}<0.01$; Fig. 4C) and IL-18 $(\mathrm{P}<0.05$; Fig. 4E) were significantly reduced, while the colonic tissue exhibited significant reductions in IL- $1 \alpha(\mathrm{P}<0.01$; Fig. $4 \mathrm{~B})$ and IL-1 $\beta$ $(\mathrm{P}<0.01$; Fig. 4D) levels, compared with the model group. In INL, compared with the model group, the serum IL-18 level was significantly reduced $(\mathrm{P}<0.05$; Fig. 4E), whereas the levels of IL- $1 \alpha(\mathrm{P}<0.01$; Fig. 4B), IL-1 $\beta$ (P<0.05; Fig. 4D) and IL-18 ( $\mathrm{P}<0.01$; Fig. 4F) in the colonic tissue were significantly reduced. In the Mes group, although there was no significant difference in the serum levels of IL- $1 \alpha$, IL- $1 \beta$ or IL-18, the colonic tissue IL- $1 \alpha(\mathrm{P}<0.01$; Fig. 4B), IL-1 $\beta$ ( $\mathrm{P}<0.01$; Fig. 4D) and IL-18 $(\mathrm{P}<0.05$; Fig. 4F) levels were significantly reduced compared with the model group.

Repair of colonic mucosal damage. Compared with the chow group, EGF and VEGF in serum and colonic tissues of the model group were decreased (Fig. 5), with significant decreases observed in the serum EGF $(\mathrm{P}<0.05$; Fig. $5 \mathrm{~A})$ and VEGF $(\mathrm{P}<0.05$; Fig. 5C), and VEGF in the colonic tissue $(\mathrm{P}<0.01$; Fig. 5D). Different doses of IN exhibited different effects in repairing colonic mucosal damage. Compared with 
A

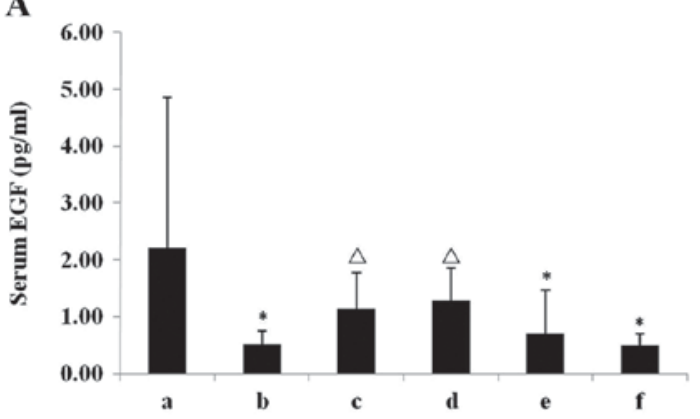

C

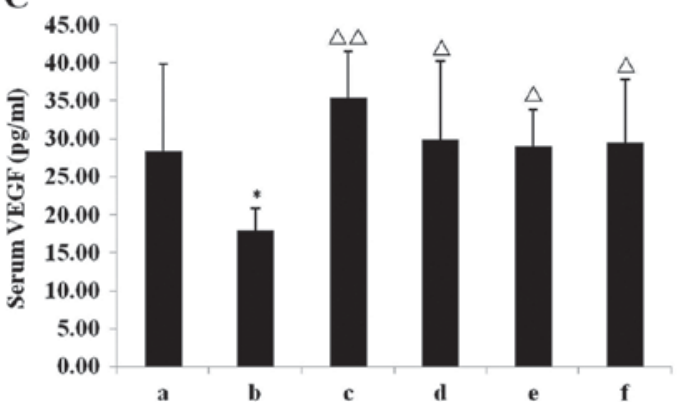

B

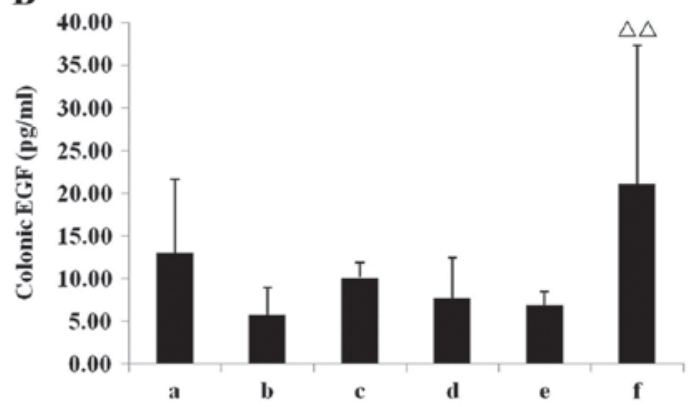

D

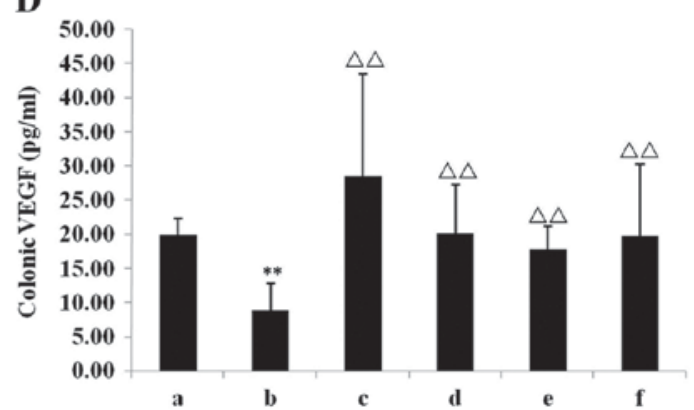

Figure 5. EGF and VEGF levels in serum and colonic tissue. (A) Serum EGF, (B) colonic EGF, (C) serum VEGF and (D) colonic VEGF levels in the six groups. ${ }^{*} \mathrm{P}<0.05,{ }^{* *} \mathrm{P}<0.01$ vs. chow group; ${ }^{\Delta} \mathrm{P}<0.05,{ }^{\Delta \Delta} \mathrm{P}<0.01$ vs. model group. Data are presented as mean \pm standard deviation. EGF, epidermal growth factor; $\mathrm{VEGF}$; vascular endothelial growth factor; a, chow group; b, model group; c, mesalazine group; d, IN high-dose group; e, IN medium-dose group; f, IN low-dose group; IN, indigo naturalis.

the model group, the serum levels of EGF $(\mathrm{P}<0.05$; Fig. $5 \mathrm{~A})$ and VEGF ( $\mathrm{P}<0.05$; Fig. $5 \mathrm{C})$, and VEGF in the colonic tissue $(\mathrm{P}<0.01$; Fig. $5 \mathrm{D})$ in the INH group were significantly increased, whereas the INM group only exhibited a significant increase in serum VEGF levels $(\mathrm{P}<0.05$; Fig. 5C) but not in serum EGF. The level of serum VEGF ( $\mathrm{P}<0.05$; Fig. $5 \mathrm{C})$, colonic EGF $(\mathrm{P}<0.01$; Fig. 5B) and VEGF $(\mathrm{P}<0.05$; Fig. 5D) in the INL group were significantly increased compared with the model group. In the Mes group, the serum levels of EGF $(\mathrm{P}<0.05$; Fig. 5A) and VEGF $(\mathrm{P}<0.01$; Fig. 5C), and VEGF in the colonic tissue $(\mathrm{P}<0.01$; Fig. $5 \mathrm{D})$, were significantly increased compared with the model group.

The quantification of immunohistochemical results indicated that the occludin content in the model group was significantly lower than the chow group ( $\mathrm{P}<0.05$; Fig. 6A). The occludin content was significantly higher in the INH $(\mathrm{P}<0.05)$ and Mes groups $(\mathrm{P}<0.01)$ compared with the model group, whereas there was no significant increase in the INL group (Fig. 6A).

The model and chow groups did not significantly differ with regard to occludin protein expression, nor did IN and Mes groups, although increasing trends were observed (Fig. 6B).

Liver injuries. To elucidate IN safety, serum AST and ALT were also assessed. In the model group, the serum AST and ALT increased, but no statistically significant difference was observed when compared with the chow group. All IN treatments demonstrated a good safety range, specifically the INH group, in which the AST and ALT were highly significantly reduced (both $\mathrm{P}<0.01$, Fig. 7) compared with the model group. In the Mes group, the serum AST was significantly reduced $(\mathrm{P}<0.05$, vs. model group; Fig. 7B), whereas no statistically significant difference was observed in ALT.

\section{Discussion}

UC is a chronic IBD (28) and its symptoms include fatigue, a constant need to defecate, nausea, diarrhoea, rectal bleeding and abdominal pain, all of which markedly affect the quality of life for the patient (29). It is a chronic lifelong condition characterized by alternating flare-ups and remission; therefore, patients with UC have a higher incidence of colon cancer than the general population (30). Male sex, young age at UC diagnosis, and extensive colitis are risk factor for the development of colon cancer (3).

Multifactorial genetic basis, environmental factors, microbiota and immune system have all been implicated to serve important roles in the pathogenesis of IBD, which is driven by an exaggerated immune response towards the gut microbiome in a genetically susceptible host $(31,32)$. According to a previous study, the number of IBD association loci is as high as 201, of which 27 loci contribute specifically to the development of UC, including innate immunity, autophagy and inflammatory response such as IL-23 receptor expression (33), other immune-mediated diseases (STAT3), and susceptibility to Mycobacterium infection (IL-12B) (34). IBD is associated with an immunological imbalance of the intestinal mucosa, primarily associated with adaptive immune system cells, which respond to self-antigens and produce chronic inflammatory conditions in patients (35). In UC, there is a marked increase in the secretion of IL-13, which is the primary interleukin responsible for inflammation and chronicity (36). Furthermore, despite Th1 involvement, patients with UC also 

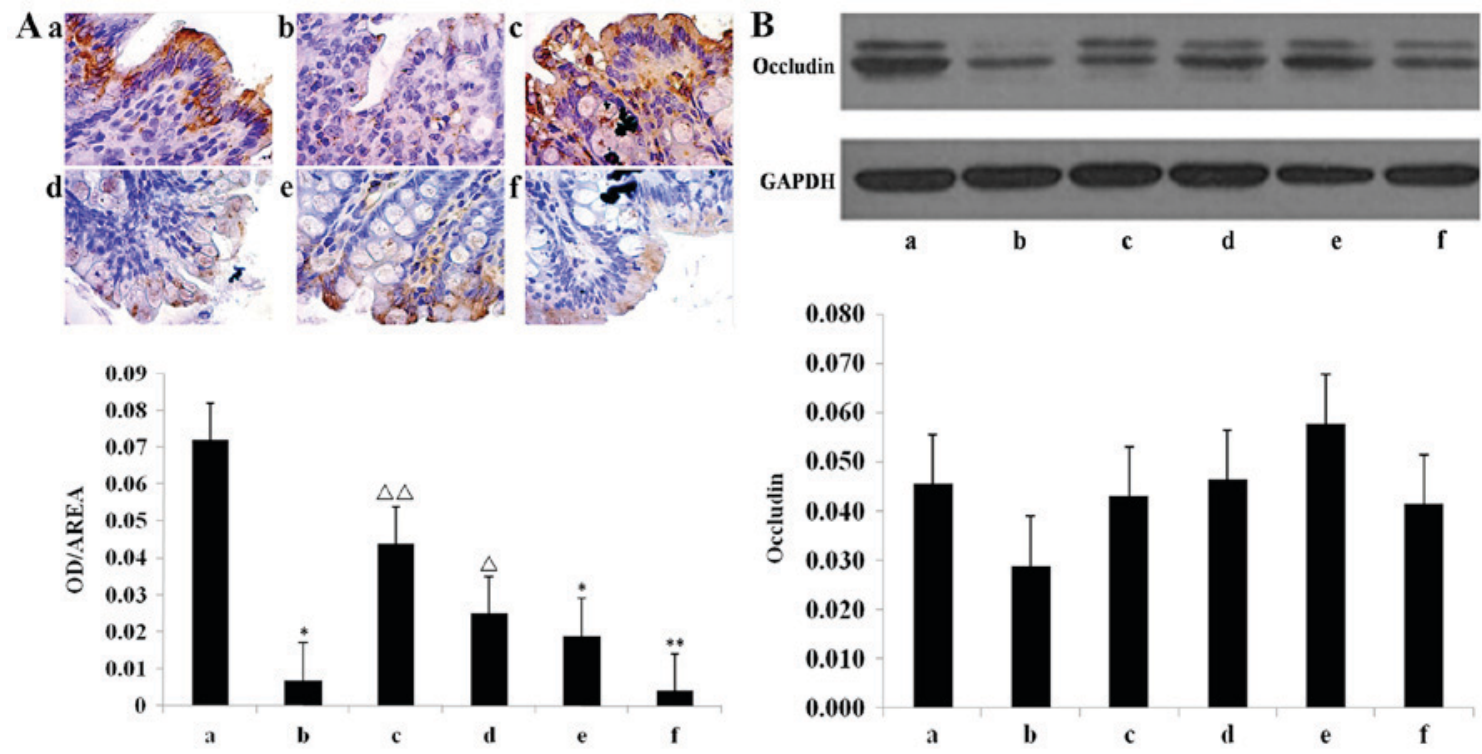

Figure 6. Expression of occludin protein. Assessed using (A) immunohistochemistry in the the (Aa) chow, (Ab) model, (Ac) mesalazine, (Ad) IN high-dose, (Ae) IN medium-dose, and (Af) IN low-dose groups, and (B) western blot analysis quantification. Thank you for your response, please add your reference to the manuscript. Make sure to add the reference to the appropriate point in the references list, carefully updating all subsequent references and in-text citations accordingly. ${ }^{*} \mathrm{P}<0.05,{ }^{* *} \mathrm{P}<0.01$ vs. chow group; ${ }^{\Delta} \mathrm{P}<0.05,{ }^{\Delta \Delta} \mathrm{P}<0.01$ vs. model group. Data are presented as mean \pm standard deviation. IN, indigo naturalis; a, chow group; b, model group; c, mesalazine group; d, IN high-dose group; e, IN medium-dose group; f, IN low-dose group; OD, optical density.

A

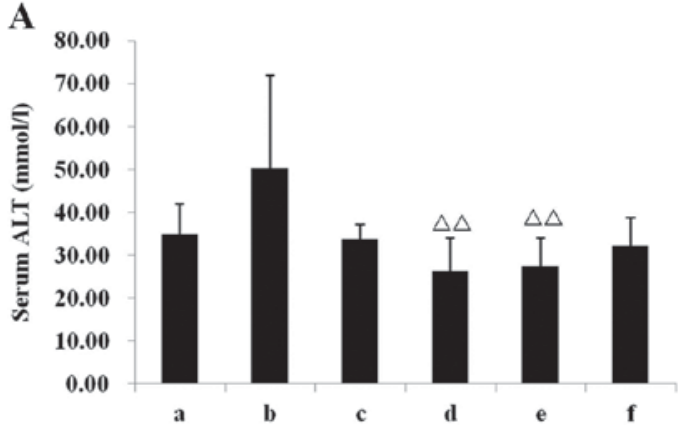

B

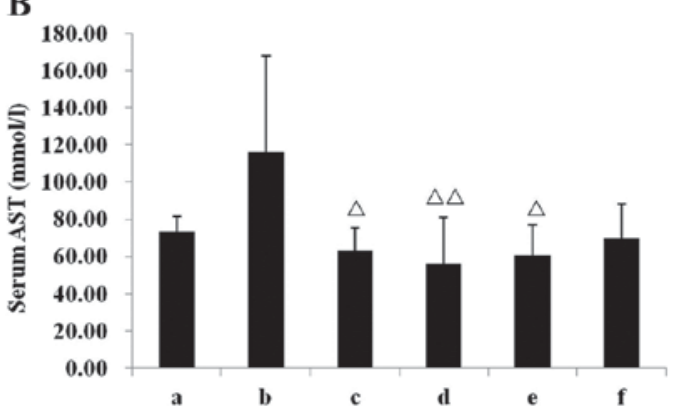

Figure 7. Effect of IN on the serum concentration of ALT and AST Concentration of serum (A) ALT and (B) AST in the six groups. ${ }^{\Delta} \mathrm{P}<0.05$, ${ }^{\Delta \Delta} \mathrm{P}<0.01$ vs. model group. Data are presented as mean \pm standard deviation. ALT, alanine transaminase; AST, aspartate transaminase; a, chow group; b, model group; c, mesalazine group; d, IN high-dose group; e, IN medium-dose group; f, IN low-dose group; IN, indigo naturalis.

present a Th2 response, with increased secretion of IL-4, IL-5 and IL-9 (36). IL-1 genes are highly upregulated in the inflamed colonic mucosa of UC (37-39). IL-1 has been demonstrated to serve a pivotal role during acute phase response, wherein the $\alpha$ form of IL- 1 is associated with exacerbations in Crohn's disease and the $\beta$ form of IL-1 is associated with UC flares during the treatment of IBD patients (40). Furthermore, mononuclear cells in the lamina propria of UC patients may secrete more IL-1 $\beta$ (41).

EGF, which consists of a class of polypeptides secreted by salivary and duodenal glands, effectively promotes mitosis and ameliorates injuries (42). EGF enema has been reported to effectively treat left hemicolonic UC (43). In preliminary studies in humans, topical EGF improved the healing of skin wounds (44) and systemic EGF helped resolve necrotizing enterocolitis in neonates (45). VEGF, a type of protein that promotes angiogenesis, may be expressed in colonic epithelial cells, endothelial cells and the muscular layer, promoting the healing and regeneration of vascular injuries (46). A marked increase in VEGF gene expression and genes encoding the receptor Flt-1 has been observed in patients with active UC and these increased VEGF levels in serum and plasma in active UC patients may reflect VEGF overexpression in intestinal inflammatory tissue (47). In addition, neutralizing anti-VEGF antibody is able to significantly ameliorate experimental UC in rats, partly by reducing excessive vascular permeability and decreasing inflammatory cell infiltration via the Src-dependent mechanism (48). Injuries of the intestinal mucosal barrier, which is constituted by colonic epithelial tight junction proteins, are important pathological factors of UC, and the tight junction transmembrane protein occludin has an important role in maintaining this barrier (49). The occludin protein content in the colonic mucosa of patients with active UC was identified to be lower than those of healthy individuals and patients with UC in the remission stage. This suggests that occludin protein serves a major role in the pathogenesis of UC (50).

Current therapies primarily involve 5-ASA agents, corticosteroids and immunosuppressive agents, of which the 5 -aminosalicylates have a potent inhibitory effect on a number of pro-inflammatory mediators released by the mucosa. These mediators include leukotrienes, IL-1 and tumour necrosis 
factor- $\alpha$ (51-53), with a response rate of 30-80\%, depending on the endpoint used (54). However, although the mechanisms of 5-ASA action are numerous and not fully understood, they are of limited benefits. As for corticosteroids, despite a number of observed benefits, patient resistance to and dependency on corticosteroids are persisting problems (55). Immunosuppressive agents such as azathioprine are beneficial but may have serious side effects (56). Therefore, novel therapeutic approaches are required (43), necessitating the identification of novel agents to counter the limitations of current UC medications. In the present study, mesalazine was used as a positive control drug in order to compare between the groups in terms of different effects, e.g., mucosal histopathology changes, activities of colon MPO, levels of inflammatory cytokines and liver injuries.

Chinese herbal medicine has been demonstrated to be effective in treating UC diseases and has a broad scope for drug safety $(57,58)$. In the present study, DSS-induced rats were used as UC animal models. The rats exhibited morphological changes in stool and blood in stool. Colonic pathology indicated marked congestion, edema, ulcer and erosion, in addition to infiltration of inflammatory cells and disappearance of crypts, replicating the onset of human UC (59). The activity of MPO, which is primarily located in inflammatory cells, specifically in the cytoplasm of neutrophils, reflects the degree of tissue infiltration of inflammatory cells and is recognized as an important indicator of inflammation (24). Colon MPO activities of DSS-induced UC rats were reduced by all doses of IN used, which is consistent with the fact that IN may significantly ameliorate DSS-induced pathological injuries. The infiltration levels of colonic inflammatory cells and severity and inflammation ranges were significantly reduced by IN treatment. Cytokines, including inflammatory factors and EGF and VEGF, in serum and colonic tissues, were detected and the concentration of IL-1 concentrations in the IN treatment group decreased. The changing trends in serum and colonic tissue levels were consistent with more prominent changes in the colon, as mononuclear cells in the lamina propria of patients with UC secrete more IL-1 $\beta$ (41). Concentrations of IL-18 in the IN treatment groups were significantly reduced, with more marked changes observed in the serum. IL-18 has been demonstrated to markedly increase in the serum of patients with UC and serum IL-18, rather than colonic mucosal IL-18, may be an effective indicator to assess the disease activity of UC (60). However, changes in serum IL-1 $\alpha$ in DSS-induced UC rats were not significant, although the levels were significantly increased in the colon. The primary reason for this may be that in DSS-induced UC rats, IL-1 $\alpha$ is predominantly produced by colonic epithelial cells, whereas IL-1 $\beta$ originates in myeloid cells (61). IN increased the expression of serum EGF, in addition to serum and colonic VEGF, but the effect on colonic EGF was not significant. Immunohistochemistry and western blot analysis indicated that a high dose of IN enhanced the occludin content in the colonic mucosa, indicating that IN may be repairing tight junction proteins, promoting EGF and VEGF expression in the colon and serum and repairing colonic mucosal damage, thereby reversing disease activity. Although dose-dependency of IN treatment was observed in some aspects, further studies are required to make this clearer.
The historical use of IN over 1,000 years attests its safety, reliability and tolerability. This was confirmed by examining liver enzyme levels in response to IN treatment. All doses of IN evaluated exhibited a good safety range, specifically the INH group, in which the AST and ALT were significantly reduced compared with the model group.

In conclusion, IN had a positive effect on experimental UC by improving the inflammation by means of regulating pro-inflammatory factors and ameliorating colonic mucosal damage by repairing tight junction proteins. The present study demonstrated that IN may be an optimal agent for UC treatment due to its anti-inflammatory and colonic mucosal damage repair properties. Further experiments should focus on the in-depth investigation of the mechanisms of IN that modulate cytokines and tight junctions, in addition to the association between intestinal flora and the inflammatory immune response.

\section{Acknowledgments}

The present study was supported by G20 Project Support, The Beijing Municipal Science and Technology Plan (grant no. Z151100003815011; The Beijing municipal Science And Technology Commission, Beijing, China) and the Youth Fund of National Science Foundation of China (grant no. 81403369; State Natural Science Funds Commission, Beijing, China).

\section{References}

1. Ordás I, Eckmann L, Talamini M, Baumgart DC and Sandborn WJ: Ulcerative colitis. Lancet 380: 1606-1619, 2012.

2. Molodecky NA, Soon IS, Rabi DM, Ghali WA, Ferris M, Chernoff G, Benchimol EI, Panaccione R, Ghosh S, Barkema HW and Kaplan GG: Increasing incidence and prevalence of the inflammatory bowel diseases with time, based on systematic review. Gastroenterology 142: 46-54.e42; quiz e30, 2012.

3. Jess T, Rungoe C and Peyrin-Biroulet L: Risk of colorectal cancer in patients with ulcerative colitis: A meta-analysis of population-based cohort studies. Clin Gastroenterol Hepatol 10: 639-645, 2012.

4. Eaden JA, Abrams KR and Mayberry JF: The risk of colorectal cancer in ulcerative colitis: A meta-analysis. Gut 48: 526-535, 2001.

5. Neovius M, Arkema EV, Blomqvist P, Ekbom A and Smedby KE: Patients with ulcerative colitis miss more days of work than the general population, even following colectomy. Gastroenterology 144: 536-543, 2013.

6. Khor B, Gardet A and Xavier RJ: Genetics and pathogenesis of inflammatory bowel disease. Nature 474: 307-317, 2011.

7. Yashiro M: Ulcerative colitis-associated colorectal cancer. World J Gastroenterol 20: 16389-16397, 2014.

8. Ording AG, Horváth-Puhó E, Erichsen R, Long MD, Baron JA, Lash TL and Sørensen HT: Five-year mortality in colorectal cancer patients with ulcerative colitis or Crohn's disease: A nationwide population-based cohort study. Inflamm Bowel Dis 19: 800-805, 2013.

9. Matsuoka H, Ikeuchi H, Uchino M, Bando T, Takesue Y, Nishigami $\mathrm{T}$ and Tomita $\mathrm{N}$ : Clinicopathological features of ulcerative colitis-associated colorectal cancer pointing to efficiency of surveillance colonoscopy in a large retrospective Japanese cohort. Int J Colorectal Dis 28: 829-834, 2013.

10. Ullman T, Odze R and Farraye FA: Diagnosis and management of dysplasia in patients with ulcerative colitis and Crohn's disease of the colon. Inflamm Bowel Dis 15: 630-638, 2009.

11. Dignass A, Eliakim R, Magro F, Maaser C, Chowers Y, Geboes K, Mantzaris G, Reinisch W, Colombel JF, Vermeire S, et al: Second European evidence-based consensus on the diagnosis and management of ulcerative colitis part 1: Definitions and diagnosis. J Crohns Colitis 6: 965-990, 2012. 
12. Daperno M, Sostegni R, Rocca R, Rigazio C, Scaglione N, Castellino F, Ercole E and Pera A: Review article: Medical treatment of severe ulcerative colitis. Aliment Pharmacol Ther 16 (Suppl 4): S7-S12, 2002.

13. Torres J, Boyapati RK, Kennedy NA, Louis E, Colombel JF and Satsangi J: Systematic review of effects of withdrawal of immunomodulators or biologic agents from patients with inflammatory bowel disease. Gastroenterology 149: 1716-1730, 2015.

14. Xiao HT, Peng J, Hu DD, Lin CY, Du B, Tsang SW, Lin ZS, Zhang XJ, Lueng FP, Han QB and Bian ZX: Qing-dai powder promotes recovery of colitis by inhibiting inflammatory responses of colonic macrophages in dextran sulfate sodium-treated mice. Chin Med 10: 29, 2015.

15. Suzuki H, Kaneko T, Mizokami Y, Narasaka T, Endo S, Matsui H, Yanaka A, Hirayama A and Hyodo I: Therapeutic efficacy of the Qing Dai in patients with intractable ulcerative colitis. World J Gastroenterol 19: 2718-2722, 2013.

16. Lin YK, See LC, Huang YH, Chang YC, Tsou TC, Lin TY and Lin NL: Efficacy and safety of Indigo naturalis extract in oil (Lindioil) in treating nail psoriasis: A randomized, observer-blind, vehicle-controlled trial. Phytomedicine 21: 1015-1020, 2014.

17. Lee MY, Liu YW, Chen MH, Wu JY, Ho HY, Wang QF and Chuang JJ: Indirubin-3'-monoxime promotes autophagic and apoptotic death in JM1 human acute lymphoblastic leukemia cells and K562 human chronic myelogenous leukemia cells. Oncol Rep 29: 2072-2078, 2013.

18. Hao Y, Nagase K, Hori K, Wang S, Kogure Y, Fukunaga K, Kashiwamura S, Yamamoto S, Nakamura S, Li J, et al: Xilei san ameliorates experimental colitis in rats by selectively degrading proinflammatory mediators and promoting mucosal repair. Evid Based Complement Alternat Med 2014: 569587, 2014.

19. Hamamoto N, Maemura K, Hirata I, Murano M, Sasaki S and Katsu K: Inhibition of dextran sulphate sodium (DSS)-induced colitis in mice by intracolonically administered antibodies against adhesion molecules (endothelial leucocyte adhesion molecule-1 (ELAM-1) or intercellular adhesion molecule-1 (ICAM-1)). Clin Exp Immunol 117: 462-468, 1999.

20. Sasaki S, Hirata I, Maemura K, Hamamoto N, Murano M, Toshina K and Katsu K: Prostaglandin E2 inhibits lesion formation in dextran sodium sulphate-induced colitis in rats and reduces the levels of mucosal inflammatory cytokines. Scand J Immunol 51: 23-28, 2000

21. Dieleman LA, Palmen MJ, Akol H, Bloemena E, Peña AS, Meuwissen SG and Van Rees EP: Chronic experimental colitis induced by dextran sulphate sodium (DSS) is characterized by Th1 and Th2 cytokines. Clin Exp Immunol 114: 385-391, 1998.

22. Laroui H, Ingersoll SA, Liu HC, Baker MT, Ayyadurai S, Charania MA, Laroui F, Yan Y, Sitaraman SV and Merlin D: Dextran sodium sulfate (DSS) induces colitis in mice by forming nano-lipocomplexes with medium-chain-length fatty acids in the colon. PLoS One 7: e32084, 2012.

23. Rashidian A, Mehrzadi S, Ghannadi AR, Mahzooni P, Sadr S and Minaiyan M: Protective effect of ginger volatile oil against acetic acid-induced colitis in rats: A light microscopic evaluation. J Integr Med 12: 115-120, 2014

24. Bradley PP, Priebat DA, Christensen RD and Rothstein G: Measurement of cutaneous inflammation: Estimation of neutrophil content with an enzyme marker. J Invest Dermatol 78 : 206-209, 1982

25. Vink EI, Yondola MA, Wu K and Hearing P: Adenovirus E4-ORF3-dependent relocalization of TIF1 $\alpha$ and TIF1 $\gamma$ relies on access to the Coiled-Coil motif. Virology 422: 317-325, 2012.

26. Gaifulina R, Maher AT, Kendall C, Nelson J, Rodriguez-Justo M, Lau K and Thomas GM: Label-free Raman spectroscopic imaging to extract morphological and chemical information from a formalin-fixed, paraffin embedded rat colon tissue section. Int J Exp Pathol 97: 337-350, 2016.

27. Shen T, Gu D, Zhu Y, Shi J, Xu D and Cao X: The value of eosinophil VCS parameters in predicting hepatotoxicity of antituberculosis drugs. Int J Lab Hematol 38: 514-519, 2016.

28. Yashiro M: Ulcerative colitis-associated colorectal cancer. World J Gastroenterol 20: 16389-16397, 2014.

29. Yarlas A, Yen L and Hodgkins P: The relationship among multiple patient-reported outcomes measures for patients with ulcerative colitis receiving treatment with MMX $®$ formulated delayed-release mesalamine. Qual Life Res 24: 671-683, 2015.

30. Abraham BP: Cancer surveillance in ulcerative colitis and Crohn's disease: New strategies. Curr Opin Gastroenterol 32: 32-37, 2016.
31. Coskun M: Intestinal epithelium in inflammatory bowel disease. Front Med (Lausanne) 1: 24, 2014.

32. Akiho H, Yokoyama A, Abe S, Nakazono Y, Murakami M, Otsuka Y, Fukawa K, Esaki M, Niina Y and Ogino H: Promising biological therapies for ulcerative colitis: A review of the literature. World J Gastrointest Pathophysiol 6: 219-227, 2015.

33. Duerr RH, Taylor KD, Brant SR, Rioux JD, Silverberg MS, Daly MJ, Steinhart AH, Abraham C, Regueiro M, Griffiths A, et al: A genome-wide association study identifies IL23R as an inflammatory bowel disease gene. Science 314: 1461-1463, 2006.

34. Bianco AM, Girardelli M and Tommasini A: Genetics of inflammatory bowel disease from multifactorial to monogenic forms. World J Gastroenterol 21: 12296-12310, 2015.

35. de Mattos BR, Garcia MP, Nogueira JB, Paiatto LN, Albuquerque CG, Souza CL, Fernandes LG, Tamashiro WM and Simioni PU: Inflammatory bowel disease: An overview of immune mechanisms and biological treatments. Mediators Inflamm 2015: 493012, 2015.

36. Liu ZJ, Yadav PK, Su JL, Wang JS and Fei K: Potential role of Th17 cells in the pathogenesis of inflammatory bowel disease. World J Gastroenterol 15: 5784-5788, 2009.

37. Yamamoto-Furusho JK, Santiago-Hernández JJ, Pérez-Hernández N, Ramírez-Fuentes S, Fragoso JM and Vargas-Alarcón G: Interleukin $1 \beta$ (IL-1B) and IL-1 antagonist receptor (IL-1RN) gene polymorphisms are associated with the genetic susceptibility and steroid dependence in patients with ulcerative colitis. J Clin Gastroenterol 45: 531-535, 2011.

38. Planell N, Lozano JJ, Mora-Buch R, Masamunt MC, Jimeno M, Ordás I, Esteller M, Ricart E, Piqué JM, Panés J and Salas A: Transcriptional analysis of the intestinal mucosa of patients with ulcerative colitis in remission reveals lasting epithelial cell alterations. Gut 62: 967-976, 2013

39. Román J, Planell N, Lozano JJ, Aceituno M, Esteller M, Pontes C, Balsa D, Merlos M, Panés J and Salas A: Evaluation of responsive gene expression as a sensitive and specific biomarker in patients with ulcerative colitis. Inflamm Bowel Dis 19: 221-229, 2013.

40. Andus T, Daig R, Vogl D, Aschenbrenner E, Lock G, Hollerbach S, Köllinger M, Schölmerich J and Gross V: Imbalance of the interleukin 1 system in colonic mucosa-association with intestinal inflammation and interleukin 1 receptor antagonist [corrected] genotype 2. Gut 41: 651-657, 1997.

41. Reinecker HC, Steffen M, Witthoeft T, Pflueger I, Schreiber S, MacDermott RP and Raedler A: Enhanced secretion of tumour necrosis factor-alpha, IL-6, and IL-1 beta by isolated lamina propria mononuclear cells from patients with ulcerative colitis and Crohn's disease. Clin Exp Immunol 94: 174-181, 1993.

42. Heitz PU, Kasper M, van Noorden S, Polak JM, Gregory H and Pearse AG: Immunohistochemical localisation of urogastrone to human duodenal and submandibular glands. Gut 19: 408-413, 1978.

43. Sinha A, Nightingale J, West KP, Berlanga-Acosta J and Playford RJ: Epidermal growth factor enemas with oral mesalamine for mild-to-moderate left-sided ulcerative colitis or proctitis. $\mathrm{N}$ Engl J Med 349: 350-357, 2003.

44. Brown GL, Nanney LB, Griffen J, Cramer AB, Yancey JM, Curtsinger LJ III, Holtzin L, Schultz GS, Jurkiewicz MJ and Lynch JB: Enhancement of wound healing by topical treatment with epidermal growth factor. N Engl J Med 321: 76-79, 1989.

45. Sullivan PB, Brueton MJ, Tabara ZB, Goodlad RA, Lee CY and Wright NA: Epidermal growth factor in necrotising enteritis. Lancet 338: 53-54, 1991.

46. Kanazawa S, Tsunoda T, Onuma E, Majima T, Kagiyama M and Kikuchi K: VEGF, basic-FGF, and TGF-beta in Crohn's disease and ulcerative colitis: A novel mechanism of chronic intestinal inflammation. Am J Gastroenterol 96: 822-828, 2001

47. Frysz-Naglak D, Fryc B, Klimacka-Nawrot E, Mazurek U, Suchecka W, Kajor M, Kurek J and Stadnicki A: Expression, localization and systemic concentration of vascular endothelial growth factor (VEGF) and its receptors in patients with ulcerative colitis. Int Immunopharmacol 11: 220-225, 2011.

48. Tolstanova G, Khomenko T, Deng X, Chen L, Tarnawski A, Ahluwalia A, Szabo S and Sandor Z: Neutralizing anti-vascular endothelial growth factor (VEGF) antibody reduces severity of experimental ulcerative colitis in rats: Direct evidence for the pathogenic role of VEGF. J Pharmacol Exp Ther 328: 749-757, 2009.

49. Al-Sadi R, Khatib K, Guo S, Ye D, Youssef M and Ma T: Occludin regulates macromolecule flux across the intestinal epithelial tight junction barrier. Am J Physiol Gastrointest Liver Physiol 300: G1054-G1064, 2011 
50. Yamamoto-Furusho JK, Mendivil EJ and Fonseca-Camarillo G: Differential expression of occludin in patients with ulcerative colitis and healthy controls. Inflamm Bowel Dis 18: E1999, 2012.

51. Nielsen $\mathrm{OH}$, Verspaget $\mathrm{HW}$ and Elmgreen J: Inhibition of intestinal macrophage chemotaxis to leukotriene B4 by sulphasalazine, olsalazine, and 5-aminosalicylic acid. Aliment Pharmacol Ther 2: 203-211, 1988.

52. Kaiser GC, Yan F and Polk DB: Mesalamine blocks tumor necrosis factor growth inhibition and nuclear factor kappaB activation in mouse colonocytes. Gastroenterology 116: 602-609, 1999.

53. Caprilli R, Cesarini M, Angelucci E and Frieri G: The long journey of salicylates in ulcerative colitis: The past and the future. J Crohns Colitis 3: 149-156, 2009.

54. Hanauer SB: Medical therapy of ulcerative colitis. Lancet 342: 412-417, 1993.

55. Gionchetti P, Rizzello F, Annese V, Armuzzi A, Biancone L, Castiglione F, Comberlato M, Cottone M, Danese S, Daperno M, et al: Use of corticosteroids and immunosuppressive drugs in inflammatory bowel disease: Clinical practice guidelines of the Italian Group for the Study of Inflammatory Bowel Disease. Dig Liver Dis pii: S1590-8658, 2017.
56. Podolsky DK: Inflammatory bowel disease. N Engl J Med 347: 417-429, 2002.

57. Wan P, Chen H, Guo Y and Bai AP: Advances in treatment of ulcerative colitis with herbs: From bench to bedside. World J Gastroenterol 20: 14099-14104, 2014.

58. Guo M, Ding S, Zhao C, Gu X, He X, Huang K, Luo Y, Liang Z, Tian $\mathrm{H}$ and $\mathrm{Xu} \mathrm{W}$ : Red ginseng and semen coicis can improve the structure of gut microbiota and relieve the symptoms of ulcerative colitis. J Ethnopharmacol 162: 7-13, 2015.

59. Perse M and Cerar A: Dextran sodium sulphate colitis mouse model: Traps and tricks. J Biomed Biotechnol 2012: 718617, 2012.

60. Wiercinska-Drapalo A, Flisiak R, Jaroszewicz J and Prokopowicz D: Plasma interleukin-18 reflects severity of ulcerative colitis. World J Gastroenterol 11: 605-608, 2005.

61. Bersudsky M, Luski L, Fishman D, White RM, Ziv-Sokolovskaya N, Dotan S, Rider P, Kaplanov I, Aychek T, Dinarello CA, et al: Non-redundant properties of IL-1 $\alpha$ and IL-1 $\beta$ during acute colon inflammation in mice. Gut 63: 598-609, 2014. 